\title{
Extended Semi-Local Convergence of Newton's Method using the Center Lipschitz Condition and the Restricted Convergence Domain
}

\author{
Ioannis K. Argyros ${ }^{1}$ and Santhosh George ${ }^{2 *}$ \\ ${ }^{1}$ Department of Mathematical Sciences, Cameron University Lawton, OK 73505, USA \\ ${ }^{2}$ Department of Mathematical and Computational Sciences, National Institute of Technology Karnataka, India-757 025 \\ ${ }^{*}$ Corresponding author E-mail: sgeorge@nitk.ac.in
}

\section{Article Info}

Keywords: Banach space, CenterLipschitz condition, Newton's method, Semi-local convergence

2010 AMS: 47H10, 49M15, 65G99,

$65 \mathrm{H} 10$

Received: 27 December 2018

Accepted: 4 February 2019

Available online: 17 June 2019

\begin{abstract}
The objective of this study is to extend the usage of Newton's method for Banach space valued operators. We use our new idea of restricted convergence domain in combination with the center Lipschitz hypothesis on the Fréchet-derivatives where the center is not necessarily the initial point. This way our semi-local convergence analysis is tighter than in earlier works (since the new majorizing function is at least as tight as the ones used before) leading to weaker criteria, better error bounds more precise information on the solution. These improvements are obtained under the same computational effort.
\end{abstract}

\section{Introduction}

Let $X, Y$ denote Banach spaces and $\Omega \subseteq X$ be a convex set. Numerous problems in diverse areas are written as an equation like

$$
F(x)=0,
$$

where $F: \Omega \longrightarrow Y$ is a twice continuously Fréchet-differentiable operator. One wishes that a solution $x_{*}$ of equation (1.1) can be found in closed form [1]-[10]. However, this is done only in special cases. This is why most researchers use iterative procedures to generate a sequence $\left\{x_{n}\right\}$ approximating $x_{*}$. The most popular iterative procedure is undoubtedly Newton's method defined for some given initial point $x_{0} \in \Omega$ by

$$
x_{n+1}=x_{n}-F^{\prime}\left(x_{n}\right)^{-1} F\left(x_{n}\right),
$$

for each $n=0,1,2, \ldots$. There is literature on convergence results for Newton's method, see $[3,8,9,10]$ and the references therein. The convergence domain of Newton's method is small in general under generalized-type Lipschitz conditions. This fact limits the applicability of Newton's method. Therefore, techniques that will enlarge the convergence domain without additional hypotheses are useful. In particular, we are motivated by the work of Ezquerro and Hernandez in [5, 6], where the center-Lipschitz on the second Fréchet-derivative was used but the center is not necessarily the starting point for Newton's method. This idea has also been used but on the first Fréchet-derivative. Using this technique in connection to majorizing functions and sequences a semi-local convergence analysis was given in [6] for the special case, when $X=Y=\mathbb{R}^{m}$, where $m$ is a positive integer. The choice of a point other than $x_{0}$ in the center-Lipschitz condition allows more flexibility in the choice of majorizing functions and sequences. Moreover, the convergence domain may be extended in some cases as it was shown in [6] for a certain class of nonlinear integral equations.

In the present study we also use the center Lipschitz condition at $x_{0}$ as well as at a point other than $x_{0}$. This way we locate a smaller domain $\Omega$ where the iterates $\left\{x_{n}\right\}$ are located. Then, the majorizing function related to the smaller domain $U_{0}$ is always at least as small as the majorizing function in $[5,6]$ derived using the set $\Omega$. We then provide a semi-local convergence analysis along the lines of the work in $[5,6]$ but with the center-Lipschitz condition on the first derivative instead of the second leading to tighter error estimates on $\left\|F^{\prime}\left(x_{n}\right)^{-1}\right\|$. This 
modification together with the usage of the new majorant function instead of the old one leads to an at least tighter semi-local convergence. Some of the advantages include weaker sufficient semi-local convergence criteria (i.e., larger convergence domain than before, tighter error estimates on the distances $\left\|x_{n+1}-x_{n}\right\|,\left\|x_{n}-x_{*}\right\|$ and more precise information on the location of the solution $x_{*}$. The interesting part of this new technique is the fact that no additional conditions are utilized since the computation of the old majorant function requires the computation of the new majorant function as a special case. Our idea can be extended in the case $F^{(i)}$ is center-Lipschitz continuous where $i \geq 2$ [2]-[4].

The lay out of the rest of the paper contains: The semi-local convergence of Newton's method in Section 2. Section 3 has the examples on which the theoretical results are tested.

\section{Semi-local convergence}

Let $\gamma \geq 0$. Define $R=\sup \left\{t \geq \gamma: U\left(x_{0}, t\right) \subseteq \Omega\right\}$. Throughout this paper $U\left(x_{0}, r\right), \bar{U}\left(x_{0}, r\right)$, stand respectively for the open and closed balls in $X$ with center at $x_{0}$ and radius $r$. We base the semi-local convergence analysis of Newton's method on the conditions $(\mathscr{A})$ :

$\left(\mathscr{A}_{0}\right)$ Operator $F: \Omega \subseteq X \longrightarrow Y$ is twice Fréchet differentiable in the Fréchet sense.

$\left(\mathscr{A}_{1}\right)$ Let $x_{0} \in \Omega$. There exist $z \in D$, and $\delta \geq 0$ such that $\left\|x_{0}-z\right\|=\delta$. Set $t_{0}=\gamma+\delta$.

$\left(\mathscr{A}_{2}\right)$ There exist operator $\Gamma_{0}=F^{\prime}\left(x_{0}\right)^{-1} \in L(Y, X), b_{1}>0$ such that $\left\|\Gamma_{0}\right\| \leq b_{1}$ and a function $g_{1}:[\gamma,+\infty) \longrightarrow[0,+\infty)$ continuous and nondecreasing such that

$$
b_{1}\left\|F^{\prime}\left(x_{0}\right)-F^{\prime}(x)\right\| \leq g_{1}\left(\left\|x_{0}-x\right\|\right)
$$

for each $x \in U\left(x_{0}, R-t_{0}\right)$. Equation $g_{1}\left(t-t_{0}\right)-1=0$ has positive solutions $t \geq t_{0}$. Denote by $\rho_{1}$ the smallest such solution. Or

$\left(\mathscr{A}_{2}^{\prime}\right)$ there exist operator $\Delta=F^{\prime}(z)^{-1} \in L(Y, X), b_{2}>0$ such that $\|\Delta\| \leq b_{2}$ and a function $g_{2}:[\gamma,+\infty) \longrightarrow[0,+\infty)$ continuous and nondecreasing such that

$$
b_{2}\left\|F^{\prime}(z)-F^{\prime}(x)\right\| \leq g_{2}(\|z-x\|)
$$

for each $x \in U\left(x_{0}, R-t_{0}\right)$. Equation $b_{2} g_{2}(t-\gamma)-1=0$ has a minimal solutions $\rho_{2} \geq \gamma$. Notice that if $g_{1}$ or $g_{2}$ are strictly increasing, then $\rho_{1}=g_{1}^{-1}\left(\frac{1}{b_{1}}\right)+t_{0}$ and $\rho_{2}=g_{2}\left(\frac{1}{b_{2}}\right)+\gamma$.

$\left(\mathscr{A}_{3}\right)$ There exists $f:[\gamma,+\infty) \longrightarrow[0,+\infty)$ twice continuously differentiable such that

$$
\left\|F^{\prime \prime}(z)\right\| \leq f^{\prime \prime}(\gamma)
$$

and

$$
\left\|\Gamma_{0} F\left(x_{0}\right)\right\| \leq-\frac{f\left(t_{0}\right)}{f^{\prime}\left(t_{0}\right)}
$$

$\left(\mathscr{A}_{4}\right) \frac{b_{1}}{1-b_{1} g_{1}(t)} \leq-\frac{1}{f^{\prime}(t)}$ for all $t \in\left[t_{0}, \rho_{1}\right]$.

$\left(\mathscr{A}_{4}^{\prime}\right) \frac{b_{2}}{1-b_{2} g_{2}(t)} \leq-\frac{1}{f^{\prime}(t)}$ for all $t \in\left[\gamma, \rho_{2}\right]$.

$\left(\mathscr{A}_{5}\right)\left\|F^{\prime \prime}(x)-F^{\prime \prime}(z)\right\| \leq f^{\prime \prime}(t)-f^{\prime \prime}(\gamma)$ for all $x \in U_{0}, t \in[\gamma, R)$, where $U_{0}=\Omega \cap U\left(x_{0}, \rho_{1}-t_{0}\right)$.

$\left(\mathscr{A}_{5}^{\prime}\right)\left\|F^{\prime \prime}(x)-F^{\prime \prime}(z)\right\| \leq f^{\prime \prime}(t)-f^{\prime \prime}(\gamma)$ for all $x \in U_{1}, t \in[\gamma, R)$, where $U_{1}=\Omega \cap U\left(x_{0}, \rho_{2}-\gamma\right)$.

(乐) $b_{1} \leq-\frac{1}{f^{\prime}\left(t_{0}\right)}$ or

$\left(\mathscr{A}_{6}^{\prime}\right) b_{2} \leq-\frac{1}{f^{\prime}\left(t_{0}\right)}$.

We shall use the majorizing Newton iteration function $f$ defined by,

$$
t_{n}=N_{f}\left(t_{n-1}\right)=t_{n-1}-\frac{f\left(t_{n-1}\right)}{f^{\prime}\left(t_{n-1}\right)} \text { for all } n=1,2, \ldots,
$$

where $t_{0}$ is given. Conditions $\left(\mathscr{A}_{0}\right),\left(\mathscr{A}_{1}\right)-\left(\mathscr{A}_{6}\right)$ or conditions $\left(\mathscr{A}_{0}\right),\left(\mathscr{A}_{1}\right),\left(\mathscr{A}_{2}^{\prime}\right),\left(\mathscr{A}_{3}\right),\left(\mathscr{A}_{4}^{\prime}\right),\left(\mathscr{A}_{5}^{\prime}\right)$ and $\left(\mathscr{A}_{6}^{\prime}\right)$ shall be called the conditions $(\mathscr{A})$.

Remark 2.1. The following conditions were used in [5]-[10] for the special case $X=Y=\mathbb{R}^{m}$ :

$\left(\mathscr{C}_{1}\right)$ There exists $z \in \Omega$ and $\delta \geq 0$ such that $\left\|x_{0}-z\right\|=\delta$ and $\left\|F^{\prime \prime}(z)\right\| \leq f_{1}^{\prime \prime}(\gamma)$.

$\left(\mathscr{C}_{2}\right)$ There exists the operator $\Gamma_{0}=F^{\prime}\left(x_{0}\right)^{-1} \in L\left(\mathbb{R}^{m}, \mathbb{R}^{m}\right)$ with $\left\|\Gamma_{0}\right\| \leq-\frac{1}{f^{\prime}\left(t_{0}\right)}$ and $\left\|\Gamma_{0} F\left(x_{0}\right)\right\| \leq-\frac{f_{1}\left(t_{0}\right)}{f_{1}^{\prime}\left(t_{0}\right)}$.

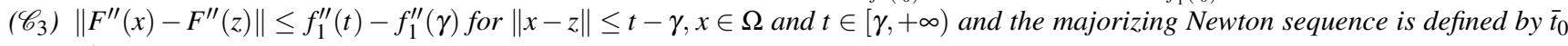
given,

$$
\bar{t}_{n}=N_{f_{1}}\left(\bar{t}_{n-1}\right)=\bar{t}_{n-1}-\frac{f_{1}\left(\bar{t}_{n-1}\right)}{f_{1}^{\prime}\left(\bar{t}_{n-1}\right)}
$$

for each $n=1,2,3, \ldots$ and $\bar{t}_{0}=t_{0}$.

Notice that $U_{0} \subseteq \Omega$ and $U_{1} \subseteq \Omega$. Therefore $f$ is at least as tight as $f_{1}$, i.e.,

and

$$
\begin{aligned}
f(t) & \leq f_{1}(t) \\
-\frac{1}{f^{\prime}(t)} & \leq-\frac{1}{f_{1}^{\prime}(t)}
\end{aligned}
$$

$$
f^{\prime \prime}(t)-f^{\prime \prime}(\gamma) \leq f_{1}^{\prime \prime}(t)-f_{1}^{\prime \prime}(\gamma)
$$


Next, we state a well known result $[9,10]$.

Lemma 2.2. Suppose that there exists a nonnegative scalar sequence $\left\{t_{n}\right\}$ majorizing a sequence $\left\{x_{n}\right\} \subseteq \Omega$. Moreover, suppose that $\lim _{n \longrightarrow+\infty} t_{n}=t_{*}$ for some $t_{*} \geq 0$. Then, there exists $x_{*} \in \Omega$ such that $\lim _{n \longrightarrow \infty} x_{n}=x_{*}$ and $\left\|x_{*}-x_{n}\right\| \leq t_{*}-t_{n}$ for each $n=0,1,2,3, \ldots$

The proof of the next two results are skipped, since these are immediately obtained from the ones in [5, 6] by using function $f$, iteration $\left\{t_{n}\right\}$, condition $(\mathscr{A})$ instead of function $f_{1}$, iterate $\left\{\bar{t}_{n}\right\}$ and conditions $\left(\mathscr{C}_{1}\right)-\left(\mathscr{C}_{3}\right)$, respectively. Moreover, these results involve solutions of scalar equations related to Newton's sequence $\left\{t_{n}\right\}$.

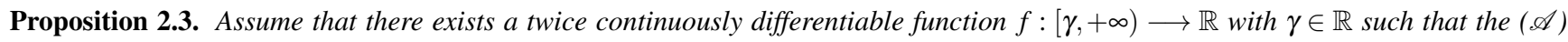
conditions are satisfied.

(1) If there exists a solution $\delta \in(\gamma,+\infty)$ of equation $f^{\prime}(t)=0$, then $\delta$ is the minimum value of $f$ in $[\gamma,+\infty)$ and $f$ is non-increasing in $\left[t_{0}, \delta\right)$.

(2) If $f(\delta) \leq 0$, then the equation $f(t)=0$ has a unique solution $t_{*}$ in $(\gamma, \delta)$ satisfying $t_{0}<t_{*}<\delta$.

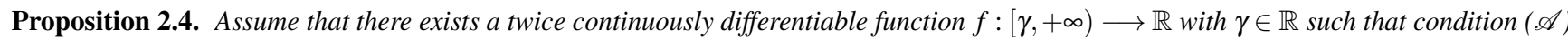
are satisfied. If there exist a solution $\delta \in[\gamma,+\infty)$ of equation $f^{\prime}(t)=0$ satisfying $f(\delta) \leq 0$, then the scalar sequence $\left\{t_{n}\right\}$ given by (2.1) is nondecreasing and converges to the minimal solution $t_{*}$ of $f(t)=0$.

Next, the semi-local convergence of Newton's method follows.

Theorem 2.5. Let $F: \Omega \subseteq X \longrightarrow Y$ be a twice continuously differentiable operator in the Fréchet sense. Assume that there exist a function $f:[\gamma,+\infty) \longrightarrow \mathbb{R}$ twice continuously differentiable with $\gamma \in \mathbb{R}$ such that conditions $(\mathscr{A})$ are satisfied and a solution $\delta \in(\gamma,+\infty)$ of equation $f^{\prime}(t)=0$ satisfying $f(\delta) \leq 0$ and $U\left(x_{0}, t_{*}-t_{0}\right) \subset \Omega$. Then, the sequence $\left\{x_{n}\right\}$ generated by Newton's method is well defined stays in $\bar{U}\left(x_{0}, t_{*}-t_{0}\right)$, and converges to a solution $x_{*} \in \bar{U}\left(x_{0}, t_{*}-t_{0}\right)$ of equation $F(x)=0$, so that

$$
\left\|x_{*}-x_{n}\right\| \leq t_{*}-t_{n} \text { for each } n=0,1,2, \ldots
$$

where sequence $\left\{t_{n}\right\}$ is given in (2.1).

Proof. We use $\left(\mathscr{A}_{2}\right)$ instead of $\left(\mathscr{C}_{3}\right)$ used in $[5,6]$ to obtain

$$
\left\|F^{\prime}\left(x_{i}\right)^{-1}\right\| \leq \frac{b_{1}}{1-b_{1} g_{1}\left(t_{i}\right)}
$$

or under $\left(\mathscr{A}_{2}^{\prime}\right)$

instead of

$$
\left\|F^{\prime}\left(x_{i}\right)^{-1}\right\| \leq \frac{b_{2}}{1-b_{2} g_{2}\left(t_{i}\right)}
$$

$$
\left\|F^{\prime}\left(x_{i}\right)^{-1}\right\| \leq-\frac{1}{f^{\prime}\left(t_{i}\right)} .
$$

Then, by $\left(\mathscr{A}_{4}\right)$ or $\left(\mathscr{A}_{4}^{\prime}\right)$ we get that the preceding estimate also holds in our setting. Using this modification, the rest of proof follows as in $[5,6]$ with sequence $\left\{t_{n}\right\}$ replacing $\left\{\bar{t}_{n}\right\}$.

The next result provides information about the location of the solution.

Proposition 2.6. Assume that the condition $(\mathscr{A})$ are satisfied. If the equation $f(t)=0$ has two solutions such that $t_{0}<t_{*} \leq t_{* *}$, then $x_{*}$ is unique in $U\left(x_{0}, t_{* *}-t_{0}\right) \cap \Omega$ provided that $t_{*}<t_{* *}$ or in $\bar{U}\left(x_{0}, t_{*}-t_{0}\right)$, provided that $t_{*}=t_{* *}$.

Proof. Simply replace $f_{1}, \bar{t}_{*}, \bar{t}_{* *}, \mathbb{R}^{m}, \mathbb{R}^{m}$ by $f, t_{*}, t_{* *}, X, Y$, respectively in [5, 6, Theorem 7].

The following error bounds are also available:

Proposition 2.7. Assume that the hypotheses of Proposition 2.6 are satisfied.

(1) For $t_{*}<t_{* *}$, suppose there exist $a_{1}>0$ and $b_{1}>0$ such that $a_{1} \leq \min \left\{\varphi(t): t \in\left[t_{0}, t_{*}\right]\right\}$ and $b_{1} \geq \max \left\{\varphi(t): t \in\left[t_{0}, t_{*}\right]\right\}$, then

$$
\frac{\left(t_{* *}-t_{*}\right) \tau^{2^{n}}}{a_{1}-\tau^{2^{n}}} \leq t_{*}-t_{n} \leq\left(t_{* *}-t_{*}\right) c^{2^{n}}
$$

for all $n=0,1,2, \ldots$ where $\varphi(t)=\frac{\left(t_{* *}-t\right) h^{\prime}(t)-h(t)}{\left(t_{*}-t\right) h^{\prime}(t)-h(t)}, f(t)=\left(t-t_{*}\right)\left(t-t_{* *}\right) h(t), h\left(t_{*}\right) \neq 0, h\left(t_{* *}\right)=0$ and $\tau=\frac{t_{*}}{t_{* *}} a_{1}$, provided that $\tau<1$ and $c<1$.

(2) For $t_{*}=t_{* *}$, suppose there exists $b_{3}>0$ such that $b_{3} \leq \min \left\{\psi(t): t \in\left[t_{0}, t_{*}\right]\right\}$, then

$$
a_{2}^{n} t_{*} \leq t_{*}-t_{n} \leq b_{3}^{n} t_{*}
$$

for all $n=0,1,2, \ldots$ provided that $a_{2}<1$ and $b_{3}<1$, where $\psi(t)=\frac{\left(t_{*}-t\right) h^{\prime}(t)-h(t)}{\left(t_{*}-t\right) h^{\prime}(t)-2 h(t)}$.

Proof. Simply replace $f_{1}, \bar{t}_{n}, \bar{t}_{*}, \bar{t}_{* *}$ by $f, t_{n}, t_{*}, t_{* *}$ in $[5,6$, Theorem 8$]$.

Remark 2.8. (i) It follows from Proposition 2.7 that the convergence order is quadratic for $t_{*}<t_{* *}$, and linear, for $t_{*}=t_{* *}$. 
(ii) The uniqueness of the solution $x_{*}$ is more precise under the new conditions. Notice that $f\left(\bar{t}_{*}\right) \leq f_{1}\left(\bar{t}_{*}\right)=0$ so $t_{*} \leq \bar{t}_{*}$. Let us suppose that for $t_{0}=0[3,5]-[10]$

$$
\begin{aligned}
& f(t)=\frac{p}{2} t^{2}-\frac{t}{b}+\frac{\eta}{b}, \\
& f_{1}(t)=\frac{q}{2} t^{2}-\frac{t}{b}+\frac{\eta}{b},
\end{aligned}
$$

then $0<p \leq q$ (provided that $2 b q \eta \leq 1$ ), we have that $t_{*} \leq \bar{t}_{*}$ and $t_{* *} \leq \bar{t}_{* *}$. Hence, the uniqueness of the solution $x_{*}$ is improved. Similar favorable comparisons are given for the lower and upper bounds given in Proposition 2.7.

(iii) The construction of function $f$ defined on $U_{0}$ as identical to the construction of function $f_{1}$ on $\Omega$ in $[5,6]$ is omitted. See also preceding case (ii) and the example in the next Section.

\section{Numerical example}

We present an example where our results apply to solve an equation but not earlier ones $[5,6]$.

Example 3.1. Let $X=Y=\mathbb{R}, \Omega=\bar{U}\left(x_{0}, 1-p\right), x_{0}=1, p \in I_{0}=\left[2-\sqrt{3}, \frac{1}{2}\right]$ and $z=x_{0}$. Define function $F$ on $\Omega$ by

$$
F(x)=\frac{x^{3}}{3}-p x+\frac{2 p}{3}
$$

Under the approach in [5, 6],

$$
\begin{gathered}
\left\|F^{\prime \prime}(x)\right\|=2\|x\| \leq 2\left(\left\|x-x_{0}\right\|+\left\|x_{0}\right\|\right) \leq 2(1-p+1)=2(2-p), \\
\left\|F^{\prime \prime}\left(x_{0}\right)-F^{\prime \prime}(x)\right\|=2\left\|x_{0}-x\right\| \leq 2, \\
b=\left\|F^{\prime}\left(x_{0}\right)^{-1}\right\|=1
\end{gathered}
$$

and

$$
\left\|F^{\prime}\left(x_{0}\right)^{-1} F\left(x_{0}\right)\right\|=\frac{1}{3}(1-p)=\eta .
$$

If polynomial $f$ (or $f_{\text {old }}$ ) satisfies $f\left(\mu_{2}\right) \leq 0$ (or $f_{\text {old }}\left(\mu_{4}\right) \leq 0$ ), then it has a negative solution and two positive solutions. In view of (3.1)-(3.4), the old function $f_{\text {old }}$ satisfying the conditions of Theorem 13 in [5, 6] is given by

$$
f_{\text {old }}(t)=\frac{1}{3} t^{3}+(2-p) t^{2}-t+\frac{1}{3}(1-p)
$$

Polynomial in (3.5) has a maximum at $t=\mu_{3}=\frac{1}{2-p-\sqrt{(2-p)^{2}+1}}<0$ and a minimum at $t=\mu_{4}=\frac{1}{2-p+\sqrt{(2-p)^{2}+1}}>0$ and $f_{\text {old }}\left(\mu_{4}\right)>$ 0 , for all $p \in I_{0}$.

Hence, the old results cannot guarantee that $\lim _{n \longrightarrow+\infty} x_{n}=x_{*}$. Under the new approach, since $g_{1}(t)=(3-p) t, U_{0}=\Omega \cap U\left(x_{0}, \frac{1}{3-p}\right)=$ $U\left(x_{0}, \frac{1}{3-p}\right), \rho_{1}=\frac{1}{3-p}$, so $U_{0}$ is a strict subset of $\Omega$ and,

$$
\left\|F^{\prime \prime}(x)\right\|=2\|x\| \leq 2\left[\left\|x-x_{0}\right\|+\left\|x_{0}\right\|\right] \leq 2\left(\frac{1}{3-p}+1\right) .
$$

Then, the new function $f$ is defined by

$$
f(t)=\frac{1}{3} t^{3}+\frac{4-p}{3-p} t^{2}-t+\frac{1}{3}(1-p)
$$

Polynomial given in (3.6) has a maximum at $t=\mu_{1}=\frac{1}{\frac{4-p}{3-p}-\sqrt{\left(\frac{4-p}{3-p}\right)^{2}+1}}<0$ and a minimum at $t=\mu_{2}=\frac{1}{\frac{4-p}{3-p}+\sqrt{\left(\frac{4-p}{3-p}\right)^{2}+1}}>0$.

Notice that $\left(\mathscr{A}_{4}\right)$ holds, if $p \in I_{0}, t \geq 0$ since it reduces to

$$
\frac{1}{1-(3-p) t} \leq-\frac{1}{t^{2}+2\left(\frac{4-p}{3-p}\right) t-1}
$$

or

$$
\frac{p^{2}-4 p+1}{3-p} \leq t
$$

or

$$
p^{2}-4 p+1 \leq 0
$$

which is true for $p \in I_{0}$. Moreover, we have that 


$$
f\left(\mu_{2}\right) \leq 0, \text { for all } p \in I_{0} .
$$

Therefore, under our approach $\lim _{n \longrightarrow \infty} x_{n}=x_{*}$. Furthermore, although the old results do not apply, we also have that for each $t, \bar{t} \in\left[0, \rho_{1}\right]$ with $t<\bar{t}, f(t) \leq f_{\text {old }}(\bar{t})$ and $f^{\prime}(t) \leq f_{\text {old }}^{\prime}(\bar{t})<0$ so

$$
-\frac{f(t)}{f^{\prime}(t)} \leq-\frac{f_{\text {old }}(\bar{t})}{f_{\text {old }}^{\prime}(\bar{t})}
$$

leading to

$$
\begin{aligned}
t_{n} & \leq \bar{t}_{n}, \\
t_{n+1}-t_{n} & \leq \bar{t}_{n+1}-\bar{t}_{n}
\end{aligned}
$$

and

$$
t_{*} \leq \bar{t}_{*} .
$$

Hence, the error bounds on $\left\|x_{n+1}-x_{n}\right\|,\left\|x_{n}-x_{*}\right\|$ are improved as well as the location of the solution.

\section{References}

[1] I. K. Argyros, S. Hilout, Weaker conditions for the convergence of Newton's method, J. Complexity, AMS, 28 (2012), 364-387.

[2] I. K. Argyros, S. Hilout, On the quadratic convergence of Newton's method under center-Lipschitz but not necessarily Lipschitz hypotheses, Math Slovaca, 63 (2013), 621-638.

[3] I. K. Argyros, A. A. Magreñán, Iterative Methods and Their Dynamics with Applications, CRC Press, New York, 2017.

[4] H. T. Davis, Introduction to Nonlinear Differential and Iintegral Equations, Dover Pub., New York, 1992.

[5] J. A. Ezquerro, D. Gonzalez, M. A. Hernández, Majorizing sequences for Newton's method from initial value problems, J. Comput. Appl. Math., 236 (2012), 2216-2238

[6] J. A. Ezquerro, M. A. Hernández, Majorizing sequences for nonlinear Fredhold-Hammerstein integral equations, Stud. Appl. Math., (2017), https://doi.org/10.1111/sapm.12200.

[7] J. M. Gutiérrez, A. A. Magreñán, N. Romero, On the semilocal convergence of Newton-Kantorovich method under center-Lipschitz conditions, Appl. Math. Comput., 221 (2013), 79-88.

[8] Kantorovich, L.V., Akilov, G.P., Functional Analysis, Pergamon Press, Oxford, 1982.

[9] L. B. Rall, Computational Solution of Nonlinear Operator equations, Robert E. Kreger Publishing Company, Michigan, 1979.

[10] T. Yamamoto, Historical developments in convergence analysis for Newton's and Newton-like methods, J. Comput. Appl. Math., 124 (2000), 1-23. 